\title{
Mitgliedschaft auseinanderbrechender Staaten in der Europäischen Union und im Euroraum
}

\author{
Michael Rötting*
}

Inhalt

A. Einleitung 420

B. Fortdauernde oder neu zu begründende Mitgliedschaft in der EU 421

I. Rechtslage bezüglich des Vereinigten Königreichs 422

1. Position der britischen Zentralregierung 423

2. Position der schottischen Regierung 423

3. Stellungnahme des EU-Kommissionspräsidenten behandelt nur den Fall der Entstehung eines neuen unabhängigen Staats 424

4. Anwendung auf den schottischen Fall 425

a) Argumente für die Anwendung der Sezessionsthese 425

(1) Parallelen zur Unabhängigkeit Algeriens von Frankreich 425

(2) Annahme der Entstehung eines neuen unabhängigen Staats in der jüngeren europäischen Geschichte zwei Mal nicht erfolgt $\quad 426$

b) Argumente für eine Anwendung der Dismembrationsthese 427

II. Rechtslage bezüglich einzelner Mitgliedstaaten des Euroraums 428

1. Katalonien als heutige spanische autonome Region 429

2. Belgien 429

3. Innerhalb des Euroraums derzeit keine Konstellation denkbar, in der zwei Nachfolgestaaten gleichzeitig eine fortbestehende EU-Mitgliedschaft evozieren könnten

C. Währungsrechtliche Konsequenzen

I. Das Vereinigte Königreich als Mitgliedstaat der EU, dessen Währung nicht der Euro ist und der rechtlich nicht zur Einführung des Euro verpflichtet ist

1. Währungsrechtliche Konsequenzen bei Verlust der Mitgliedschaft in der EU

2. Währungsrechtliche Konsequenzen bei einem Wiedereintritt in die EU nach zwischenzeitlichem Ausscheiden

3. Währungsrechtliche Konsequenzen einer fortbestehenden Mitgliedschaft eines verkleinerten Vereinigten Königreichs in der EU

* Bundesbankoberrat Dr. Michael Rötting, LL.M. Eur. ist im Zentralbereich Recht der Deutschen Bundesbank zuständig für Fragen des Europarechts, institutionelle Fragen im Zusammenhang mit dem Eurosystem und dem Europäischen System der Zentralbanken, Staatsrecht und Völkerrecht. Die in diesem Artikel vertretenen Aussagen geben ausschließlich die persönliche Meinung des Autors wieder. Sie dürfen in keiner Weise der Deutschen Bundesbank zugerechnet werden. 
4. Währungsrechtliche Konsequenzen einer fortbestehenden Mitgliedschaft beider Nachfolgestaaten des bisherigen Vereinigten Königreichs in der EU

a) Anpassung der Modalitäten der Mitgliedschaft 433

b) Eintritt in eine „Sterling-Union“

II. Zerfallende Mitgliedstaaten des Euroraums oder sich unabhängig erklärende Teilregionen von Mitgliedstaaten des Euroraums

1. Verhandlungsbasierte Lösung für neue Entität wahrscheinlich 436

a) Mitgliedschaftsrechte in der EU 436

b) Währungsrechtliche Fragen 437

c) Rechtfertigung einer Andersbehandlung von Mitgliedstaaten des Euroraums und von neuen unabhängigen Staaten, die den Euro bislang verwendet haben?

2. Auswirkungen auf verkleinerte Rumpfstaaten

3. Auswirkungen des Ausscheidens eines EZB-Ratsmitglieds oder dessen Unvermögens, an einer Abstimmung teilzunehmen, auf die Beschlussorgane der EZB

\section{A. Einleitung}

Unabhängigkeitsbestrebungen innerhalb von Mitgliedstaaten der Europäischen Union stellen mit Blick auf die EU-Mitgliedschaft der möglicherweise neu oder wieder entstehenden Staaten eine rechtliche Herausforderung dar, die mangels vergleichbarer Fälle in der Vergangenheit ohne einen eindeutigen Rückgriff auf frühere Fallgestaltungen bewältigt werden muss. Für Mitgliedstaaten, deren Währung der Euro ist, stellt sich die Frage der währungsrechtlichen Konsequenzen vor dem Hintergrund der ausschließlichen währungspolitischen Kompetenz der Union gemäß Art. 3 Abs. 1 lit. c) AEUV mit besonderer Dringlichkeit.

In der spanischen autonomen Region Katalonien etwa existieren Unabhängigkeitsbestrebungen, die wesentlich durch die offenen Fragen nach einer EU-Mitgliedschaft eines unabhängigen Kataloniens und dem für diesen Staat künftig geltenden währungsrechtlichen Regime beeinflusst werden könnten. In Katalonien erfolgte aktuell eine Ankündigung der katalonischen Regierung, am 9. November 2014 ein Unabhängigkeitsreferendum durchführen zu wollen. Der katalanische Ministerpräsident Mas hat am 21. September 2014 auf der Grundlage eines vom katalanischen Regionalparlament am 19. September 2014 verabschiedeten Gesetzes angekündigt, per Dekret die Durchführung des Referendums anzuordnen. Hiergegen wehrt sich der spanische Zentralstaat mit allen zur Verfügung stehenden juristischen und politischen Mitteln. Insbesondere ist zu erwarten, dass die spanische Zentralregierung das Dekret vor dem spanischen Verfassungsgericht anfechten wird. Daher ist die tatsächliche Durchführung und Legitimität dieses Referendums derzeit noch fraglich.

Allerdings stellt sich auch bei Staaten, die sich durch ein Opt-out der rechtlichen Verpflichtung zur Einführung des Euro beim Vorliegen der Beitrittsvoraussetzungen entzogen 
haben - dies betrifft unter den gegenwärtig 28 Mitgliedstaaten der Union nur das Vereinigte Königreich und Dänemark - die Frage, welches währungsrechtliche Regime in den Staaten gelten würde, die aus einer erlangten staatlichen Unabhängigkeit eines Teilgebiets eines bisherigen Mitgliedstaats hervorgehen. Diese Frage ist nach der Durchführung eines Referendums über die staatliche Unabhängigkeit Schottlands am 18. September 2014, in dem die Stimmberechtigten mit einer Mehrheit von $55 \%$ für einen Verbleib Schottlands im Vereinigten Königreich votiert haben, zwar nicht mehr von unmittelbarer aktueller Relevanz. Der in Spanien zu beobachtende Widerstand der Zentralregierung schon gegen die Durchführung eines Referendums fehlte dabei im Vereinigten Königreich, da das schottische Referendum entsprechend einer Vereinbarung der schottischen Regierung mit der Regierung des Vereinigten Königreichs durchgeführt wurde.

Auch wenn das schottische Volk sich im Referendum vom 18. September 2014 für einen Verbleib Schottlands im Vereinigten Königreich und damit indirekt auch hinsichtlich des währungsrechtlichen Regimes für den Fortbestand des status quo entschieden hat, lassen sich aus einer Betrachtung der Folgefragen, die hinsichtlich der Mitgliedschaft in der Europäischen Union und hinsichtlich des anwendbaren Währungsregimes im Fall eines Erfolges des Referendums ergeben hätten, möglicherweise - und unter Berücksichtigung der spezifischen Besonderheiten des schottischen Falls - Schlussfolgerungen auch für andere Mitgliedstaaten der Europäischen Union ziehen. Dies hat über den katalonischen Fall hinausgehende Bedeutung.

In Katalonien stellt sich gerade die Frage der Legitimität des eventuellen Referendums vor dem Hintergrund der spanischen Verfassung. In anderen Mitgliedstaaten des Euroraums, in denen teilweise Unabhängigkeitsbestrebungen von Teilregionen bestehen, so etwa in Belgien oder in Italien, besteht zwar keine vergleichbare zeitliche Dringlichkeit zur Lösung der mit einem konkreten Unabhängigkeitsreferendum verbundenen Rechtsfragen. Allerdings zeigen diese Konstellationen, dass einer Beschäftigung insbesondere mit Fragen des währungsrechtlichen Regimes durchaus praktische Bedeutung zukommt.

\section{B. Fortdauernde oder neu zu begründende Mitgliedschaft in der EU}

Als Vorfrage des währungsrechtlichen Regimes in einem unabhängigen Staat, der aus einem Gebietsteil eines Mitgliedstaats der Europäischen Union hervorgegangen ist, ist die Frage nach einer fortdauernden oder neu zu begründenden Mitgliedschaft in der Europäischen Union anzusehen. Denn Art. 3 Abs. 1 lit. c) AEUV überträgt die währungspolitische Kompetenz für die Mitgliedstaaten, deren Währung der Euro ist, ausschließlich auf die Unionsebene. Das Rechtsregime zur Währungspolitik im zweiten bis fünften Kapitel des VIII. Titels des AEUV (Art. $127 \mathrm{ff}$. AEUV) gehört somit zum acquis communautaire, dem gemeinsamen Rechtsbestand der Union. Europäische Staaten, die Mitgliedstaaten der Europäischen Union werden möchten, sind genau wie die schon bestehenden Mitgliedstaaten zur vollständigen Übernahme des acquis rechtlich verpflichtet, soweit es ihnen nicht gelingt, durch ein einvernehmlich vereinbartes Opt-out im Einzelfall von einer Verpflichtung abweichen zu dürfen. ${ }^{1}$

1 Cremer, in: Calliess/Ruffert (Hrsg.), EUV/AEUV, 4. Aufl. 2011, Art. 49 EUV, Rdnr. 6. 
Die Erfüllung dieser Verpflichtung kann gegenüber Mitgliedstaaten durch die EU-Kommission in einem Vertragsverletzungsverfahren gemäß Art. 258 AEUV eingefordert werden; gegenüber Beitrittskandidaten kann sie durch eine entsprechende Herauszögerung der Beitrittsverhandlungen indirekt sanktioniert werden. Für bestehende EU-Mitgliedstaaten, die anders als das Vereinigte Königreich und Dänemark kein währungspolitisches Optout vereinbart haben, besteht daher jedenfalls rechtlich eine Verpflichtung zur Einführung des Euro bei Erfüllung der Konvergenzkriterien.

Diesem rechtlichen Befund steht auch nicht die faktische Beobachtung entgegen, dass die EU-Kommission jedenfalls bislang gegen Mitgliedstaaten noch kein Vertragsverletzungsverfahren eingeleitet hat, ${ }^{2}$ die sich mit ihrem Beitrittsvertrag zwar rechtlich zur Einführung des Euro verpflichtet haben, aber innerstaatliche Rechtsregeln erlassen haben, die eine Bejahung der in Art. 140 AEUV enthaltenen Konvergenzkriterien verhindern. Dies gilt aktuell etwa für den Fall des Königreichs Schweden, das die ökonomischen Konvergenzkriterien zur Einführung des Euro in der Vergangenheit durchaus erfüllt hat, aber durch den Erlass inkompatibler innerstaatlicher Rechtsregeln eine Situation herbeigeführt hat, in der keine Beitrittsfähigkeit bejaht werden und folglich die für Schweden geltende Ausnahmeregelung nicht gemäß Art. 140 Abs. 2 AEUV vom Rat aufgehoben werden konnte. Diese Entwicklung wurde bislang seitens der EU-Kommission toleriert, obwohl sich Schweden anders als das Vereinigte Königreich und Dänemark durch seinen Beitrittsvertrag zur Einführung des Euro bei Vorliegen der Konvergenzkriterien rechtlich verpflichtet hat.

\section{Rechtslage bezüglich des Vereinigten Königreichs}

Der dargestellte Befund eines bestehenden Opt-outs des Vereinigten Königreichs bezüglich der Einführung des Euro im Rahmen des acquis hätte hinsichtlich der Bewertung des währungsrechtlichen Regimes in einem unabhängigen Schottland und in einem verkleinerten Vereinigten Königreich von England, Wales und Nordirland zu unterschiedlichen Ergebnissen geführt, die davon abhängig gewesen wären, wie die staatliche Trennung beider Teile des bisherigen Vereinigten Königreichs völker- und unionsrechtlich zu bewerten gewesen wäre. Sowohl die Zentralregierung des Vereinigten Königreichs als auch die schottische Regierung haben im Vorfeld des gescheiterten Unabhängigkeitsreferendums wissenschaftlichen Rat eingeholt, um die wirtschaftlichen und rechtlichen Konsequenzen einer schottischen Unabhängigkeit besser evaluieren zu können.

2 Der EuGH billigt der EU-Kommission ausdrücklich ein Ermessen zur Verfahrenseinleitung zu und lehnt es, anders als Teile der Literatur, ab, die EU-Kommission zur Einleitung eines Verfahrens in allen ihr bekannt werdenden Vertragsverletzungsfällen zu verpflichten. EuGH, Rs. C-72/90, Asia Motor France/Kommission, Slg. 1990, I-2181, Rdnr. 13; a.A. aus der Literatur etwa Ortlepp, Das Vertragsverletzungsverfahren als Instrument zur Sicherung der Legalität im Europäischen Gemeinschaftsrecht, 1987, S. 76 f.; Däubler, Die Klage der EWG-Kommission gegen einen Mitgliedstaat, NJW 1968, S. 329; Mertens de Wilmars/Veroughstraete, Proceedings against member states for failure to fulfil their obligations, CMLR 1970, S. 403. 


\section{Position der britischen Zentralregierung}

Die Regierung des Vereinigten Königreichs ging politisch davon aus, dass Schottland nach Erlangung der Unabhängigkeit vom Vereinigten Königreich ein neuer Staat geworden wäre. Das Vereinigte Königreich selbst dagegen hätte nach dieser Ansicht zwar in territorial und hinsichtlich seiner Bevölkerungsgröße verkleinerter Form, jedoch rechtlich kontinuierlich fortbestanden. Es hätte deshalb unverändert die Mitgliedschaft des Vereinigten Königreichs in den Vereinten Nationen, der Europäischen Union und anderen internationalen Organisationen fortzuführen beansprucht. ${ }^{3}$ Insbesondere hätte das verkleinerte Vereinigte Königreich den permanenten Sicherheitsratssitz des Vereinigten Königreichs für sich beansprucht und wäre von einer fortdauernden EU-Mitgliedschaft ausgegangen, deren Bedingungen, besonders hinsichtlich des Opt-outs bezüglich der Verpflichtung zur Einführung des Euro, unverändert hätten fortbestehen sollen.

Ein unabhängiger schottischer Staat hätte nach dieser Auffassung die Mitgliedschaft in internationalen Organisationen neu begründen ${ }^{4}$ und hinsichtlich der EU-Mitgliedschaft das von Art. 49 EUV hierfür vorgesehene Verfahren beschreiten müssen.

\section{Position der schottischen Regierung}

Die schottische Regierung hingegen wies in der Vorbereitungszeit des Referendums darauf hin, dass Schottland nach einem erfolgreichen Unabhängigkeitsreferendum erst in einen Verhandlungsprozess mit dem Vereinigten Königreich über die Modalitäten der Unabhängigkeit eingetreten wäre und in dieser Phase, aus der noch bestehenden EU-Mitgliedschaft als Teil des Vereinigten Königreichs heraus, über eine eigenständige EU-Mitgliedschaft hätte verhandeln können, sodass ein unabhängiges Schottland nahtlos Mitglied der Europäischen Union hätte bleiben können. ${ }^{5}$

Hierzu hätten allerdings die Beitrittsverhandlungen zeitgleich mit der Erlangung der staatlichen Unabhängigkeit abgeschlossen sein oder die Erlangung der Unabhängigkeit bis zum Abschluss der Beitrittsverhandlungen aufgeschoben werden müssen. Dabei unterstellten die schottische Regierung und die diese Argumentation vorbringenden Autoren, ${ }^{6}$ dass die EU in einem Beitrittsverfahren bereits vor Erlangung der staatlichen Unabhängigkeit mit Schottland in Verhandlungen hätte eintreten können. Dies setzt jedoch eine sehr großzügige Interpretation des von Art. 49 UAbs. 1 Satz 1 EUV als Beitrittskriterium vor-

3 HM Government, Scotland analysis: Devolution and the implications of Scottish independence, Februar 2013, Kap. 3, S. 45, „EU Membership“, 1. Spiegelstrich, sowie Executive Summary, S. 7, Pkt. xiv.

4 Ibid., Kap. 3, S. 45, „EU Membership“, 2. Spiegelstrich.

5 Scottish Government, „Scotland's Future“v. 26.11.2013, http://scotreferendum.com/(1.10.2014), S. 220.

6 Etwa Harvey, Secession and Dissolution in European Union Member States: A Prospective Analysis of the Consequences for European Union Membership, ZEuS 2013, S. 403. 
ausgesetzten Staatlichkeitskriteriums ${ }^{7}$ voraus. Darüber hinaus hätte der von der schottischen Regierung für die Beitrittsverhandlungen anberaumte Zeitraum von nur 18 Monaten zwischen dem Unabhängigkeitsreferendum und dem Wirksamwerden der Unabhängigkeit $^{8}$ dazu geführt, dass es Schottland hätte gelingen müssen, die kürzesten Beitrittsverhandlungen in der Geschichte der Europäischen Union zu führen. Der von Schottland angeregte Weg einer Vertragsänderung nach Art. 48 EUV statt eines Beitrittsverfahrens nach Art. $49 \mathrm{EUV}^{9}$ hätte sich voraussichtlich gleichfalls nicht als schnellerer Weg beschreiten lassen, wenn man bedenkt, dass Vertragsänderungen, neben der vorausgesetzten Einstimmigkeit ${ }^{10}$ und Ratifikation in allen Mitgliedstaaten, in einigen Mitgliedstaaten sogar einem obligatorischen oder fakultativen Referendum unterworfen sind.

\section{Stellungnahme des EU-Kommissionspräsidenten behandelt nur den Fall der Entstehung eines neuen unabhängigen Staats}

Den Fall des Entstehens eines neuen unabhängigen Staates behandelt auch der Brief von EU-Kommissionspräsident Barroso an Lord Tugendhat, den Acting Chairman des House of Lords vom 10. Dezember 2012, ${ }^{11}$ in dem der EU-Kommissionspräsident mit Blick auf die seitens des House of Lords am 29. Oktober 2012 an die Kommission gerichtete Anfrage zu einer eventuellen schottischen Unabhängigkeit zwar antwortet, dass es nicht die Rolle der EU-Kommission sei,

„to express a position on questions of internal organisation related to the constitutional arrangements of a particular Member State“.

Dennoch stellt der EU-Kommissionspräsident unter Berufung auf frühere Äußerungen fest, dass die Unionsverträge lediglich in den EU-Mitgliedstaaten gelten, die diesen zugestimmt und sie ratifiziert haben.

„If part of the territory of a Member State would cease to be part of that state because it were to become a new independent state, the Treaties would no longer apply to that territory. In other words, a new independent state would, by the fact of its independence, become a third country with respect to the EU and the Treaties would no longer apply on its territory." ${ }^{12}$

7 Zum Staatlichkeitskriterium Rötting, Das verfassungsrechtliche Beitrittsverfahren zur Europäischen Union, 2009, S. 93; hier noch ausdrücklich den Beitritt von Untergliederungen eines Staats, denen keine eigene Staatsqualität zukommt, ausschließend. Eine extensive Auslegung des Kriteriums „Staat" für die Aufnahme von Beitrittsverhandlungen ist hingegen bei einem im Entstehen begriffenen Staat, dem dann auch nach der Erlangung der Unabhängigkeit eigene Staatsqualität zukäme, de facto zu erwarten, sofern der politische Wille der Verhandlungsparteien besteht, ein Ausscheiden eines unabhängig werdenden Teilgebiets eines bisherigen Mitgliedstaats aus der Union zu verhindern.

8 Scottish Government, (Fn. 5), S. 220.

9 Ibid., S. 221.

10 Auf die Schwierigkeit der Erzielung einer solchen Einstimmigkeit weist am hypothetischen Beispiel einer Auflösung Belgiens, die wegen der Verortung wichtiger EU-Organe in Belgien eine Lösung erfordere, die über rein rechtliche Erwägungen hinausgeht, Harvey, (Fn. 6), S. 444 f. hin.

11 Ref. Ares(2012) 1469619 - 10/12/2012; BARROSO (2012) 1300171.

12 Ref. Ares(2012) 1469619 - 10/12/2012; BARROSO (2012) 1300171, Hervorhebung nicht im Original. 


\section{Anwendung auf den schottischen Fall}

Die - nach dem Votum der schottischen Wahlberechtigten für einen Verbleib Schottlands im Vereinigten Königreich hypothetische - Anwendung der Stellungnahme des EU-Kommissionspräsidenten auf den schottischen Fall impliziert allerdings zwei Voraussetzungen, nämlich zunächst, dass Schottland ein neuer unabhängiger Staat geworden wäre und sodann, dass die Unabhängigkeit vom Vereinigten Königreich völkerrechtlich wirksam geworden wäre, da in Schottland in einer Übergangsphase hin zur Unabhängigkeit jedenfalls so lange die Unionsverträge hätten Anwendung finden müssen, wie Schottland noch Teil des Vereinigten Königreichs geblieben wäre.

\section{a) Argumente für die Anwendung der Sezessionsthese}

Die Annahme der britischen Zentralregierung, dass ein verkleinertes Vereinigtes Königreich als solches rechtlich kontinuierlich im Falle eines Erfolgs des Unabhängigkeitsreferendums fortbestanden hätte, während Schottland ein neuer unabhängiger Staat geworden wäre, stützt sich auf eine fundierte Analyse der bisherigen völkerrechtlichen und unionsrechtlichen Praxis der Staatennachfolge mit direktem Bezug auf das Vereinigte Königreich, so insbesondere mit Bezug auf das Ausscheiden des Territoriums der heutigen Republik Irland aus dem Vereinigten Königreich im Jahre 1922, bei dem das durch die irische Unabhängigkeit verkleinerte Vereinigte Königreich seine Mitgliedschaft in internationalen Organisationen unverändert fortgesetzt hat. ${ }^{13} \mathrm{Da}$ das durch ein Ausscheiden Schottlands verkleinerte Vereinigte Königreich mit dem bisherigen Vereinigten Königreich subjektidentisch fortbestünde, läge nach dieser Ansicht an sich kein Fall der Staatennachfolge vor. Fragen der Staatennachfolge in völkerrechtliche Verträge hätten sich daher nur für das unabhängige Schottland gestellt.

\section{(1) Parallelen zur Unabhängigkeit Algeriens von Frankreich}

Hinsichtlich einer unionsrechtlichen Bewertung lässt sich diese Position durch eine Betrachtung der Unabhängigkeit Algeriens von Frankreich, dem einzigen vergleichbaren praktischen Fall in der Geschichte der Europäischen Union, untermauern. ${ }^{14}$ Algerien war vor seiner Unabhängigkeit Teil des französischen Kernlandes („France métropolitaine“) und gerade keine französische Kolonie; das Territorium des heutigen Algerien war dementsprechend integraler Teil der damaligen Europäischen Wirtschaftsgemeinschaft. Trotz der Loslösung Algeriens von Frankreich hat Frankreich seine Mitgliedschaftsrechte in der Europäischen Wirtschaftsgemeinschaft unverändert fortgesetzt. Algerien als neuer unabhängiger Staat hat zwar weder beansprucht, französische staatliche Kontinuität fortzusetzen, noch eine eigene EWG-Mitgliedschaft beantragt, allerdings wurde algerischen Waren für eine Übergangsfrist bis zum Abschluss einer vertraglichen Regelung die Warenver-

13 Crawford/Boyle, Opinion: Referendum on the Independence of Scotland - International Law Aspects, Annex A zu HM Government, Scotland analysis, (Fn. 3), S. 82, Rdnr. 65.

14 Nur im Ergebnis gleich hierzu ibid., S. 78, Rdnr. 53, die Algerien trotz der Integration ins französische Kernland als „colonial or quasi-colonial case“ einordnen. 
kehrsfreiheit weiter eingeräumt. ${ }^{15}$ Der EuGH hat im Jahre 1973 vertreten, dass Algerien „zwar am 1. Juli 1962 seine Unabhängigkeit erlangt“ habe, aber „erst aufgrund der Verordnung Nr. 109/65/EWG des Rates vom 30. Juni 1965 aufgehört habe, Teil des Hoheitsgebiets der Gemeinschaft zu sein“. ${ }^{16}$ Algerischstämmige Bürger konnten durch Optieren für eine französische Staatsangehörigkeit zudem kontinuierlich die Freizügigkeitsrechte in der Europäischen Wirtschaftsgemeinschaft beanspruchen. Vertreter des neuen algerischen Staates erhielten allerdings zu keiner Zeit eine Repräsentanz in den Organen der Gemeinschaft; eine Mitgliedschaft Algeriens in der Gemeinschaft schied zudem von vornherein aus, da es sich bei Algerien nicht um einen europäischen Staat handelte. Die Eigenschaft des Europäischseins ist seit Gründung der EWG Beitrittsvoraussetzung. ${ }^{17}$

(2) Annahme der Entstehung eines neuen unabhängigen Staats in der jüngeren europäischen Geschichte zwei Mal nicht erfolgt

Allerdings bestehen in der jüngeren europäischen Geschichte zumindest zwei Fälle einer vollständigen Staatendismembration, in denen die Rechtspraxis keinen eindeutigen Nachfolgestaat identifiziert hat: der Zerfall der Tschechoslowakei (CSFR) in die Tschechische Republik und in die Slowakei sowie der Zerfall Jugoslawiens. Der Zerfall der CSFR unterscheidet sich insofern von sonstigen Fällen der Staatennachfolge, als die Tschechische Republik und die Slowakei im Rahmen ihrer einvernehmlichen Trennung vertraglich vereinbart hatten, dass keiner der beiden Staaten alleine die Rechtsnachfolge der CSFR beanspruchen solle. ${ }^{18}$ Daher stellte sich in diesem Fall die Frage nicht, ob die Tschechische Republik als größerer der beiden Staaten die alleinige Staatennachfolge erfolgreich hätte beanspruchen können. Beim Zerfall Jugoslawiens wurde die vom verbleibenden Rumpfstaat Serbien-Montenegro beanspruchte Staatennachfolge Jugoslawiens von den anderen aus Jugoslawien hervorgegangenen Staaten bestritten und in völkerrechtlichen Organisationen, insbesondere in den Vereinten Nationen, nicht anerkannt, sodass Serbien-Montenegro zur Wahrnehmung seiner Mitgliedschaftsrechte nach einer Zeit eine Neumitgliedschaft in den Vereinten Nationen beantragte. ${ }^{19}$ Die Annahme der britischen Regierung, ein verkleinertes Vereinigte Königreich wäre als fortbestehender Staat in alle Rechtspositionen des bisherigen Vereinigten Königreichs hineingewachsen, bleibt daher mit Zweifeln behaftet, ob in der Rechtspraxis, insbesondere hinsichtlich der Frage der Mitgliedschaft in der Europäischen Union und ihrer Modalitäten, die britische Position auch durchsetzbar gewesen wäre.

15 Zimmermann, Staatennachfolge in völkerrechtliche Verträge, 2000, S. 676.

16 EuGH, Rs. 110/73, Fiege/Caisse Régionale d'Assurance Maladie de Strasbourg, Slg. 1973, 1001, 1011.

17 Hierzu Rötting, (Fn. 7), S. 94 ff.

18 Vgl. Zimmermann, (Fn. 15), S. 113; siehe auch Art. 1 des Verfassungsgesetzes über den Untergang der Tschechischen und Slowakischen Republik v. 25.11.1992.

19 Ausführlich zur Rechtspraxis hinsichtlich der Frage der völkerrechtlichen Identität SerbienMontenegros mit Jugoslawien siehe Zimmermann, (Fn. 15), S. 98-112, zur Mitgliedschaft in den Vereinten Nationen vor allem S. 108 und 110. 


\section{b) Argumente für eine Anwendung der Dismembrationsthese}

Denkbar wäre es im schottischen Fall insbesondere auch gewesen, in Anbetracht des Fortbestehens des schottischen Territoriums und einiger wichtiger schottischer Institutionen nach dem Abschluss der Unionsverträge mit England, eine Unabhängigkeit Schottlands als Auflösung dieser Unionsverträge von 1707 zwischen dem Königreich England und dem Königreich Schottland zu verstehen, welche zur Entstehung des Vereinigten Königreichs geführt haben. Hätte sich diese Rechtsauffassung in der Praxis durchgesetzt, so hätte ein Erfolg des schottischen Unabhängigkeitsreferendums zum Untergang des Vereinigten Königreichs mit zu klärenden völkerrechtlichen Konsequenzen hinsichtlich der Staatennachfolge in völkerrechtliche Verträge, insbesondere mit Blick auf die Mitgliedschaft des Vereinigten Königreichs in den Vereinten Nationen und in der Europäischen Union, geführt.

Da die Mitgliedschaft des Vereinigten Königreichs in der damaligen Europäischen Gemeinschaft durch den Beitrittsvertrag von 1973 als einem völkerrechtlichen Vertrag des Nationalstaats Vereinigtes Königreich mit den anderen damaligen Mitgliedstaaten der Europäischen Gemeinschaft zu Stande gekommen ist, müsste man annehmen, dass aus der Auflösung der schottisch-englischen Union zumindest für eine juristische Sekunde die getrennten Königreiche England und Schottland entstehen würden - wobei sodann faktisch anzunehmen ist, dass das Königreich England sich erneut mit Wales und Nordirland zu einem - allerdings neuen - Vereinigten Königreich verbunden hätte, während das Königreich Schottland entsprechend dem Selbstbestimmungsrecht des schottischen Volkes eine republikanische Regierungsform eingeführt hätte oder, wie vor dem Unabhängigkeitsreferendum von der schottischen Regierung gewünscht, ein eigenständiges Königreich innerhalb des Commonwealth hätte werden können.

Die Frage der EU-Mitgliedschaft der beiden Nachfolgestaaten hätte sich bei einem solchen Ergebnis nur einheitlich behandeln lassen. Denkbar wäre es daher gewesen, dass die Mitgliedschaft beider Nachfolgestaaten in der EU erloschen und bei einem entsprechenden Interesse am Fortbestehen der Mitgliedschaft im Rahmen eines Beitrittsverfahrens, wie es Art. 49 EUV vorsieht, neu hätte beantragt werden müssen. Dieser Fall wäre damit der völkerrechtlichen Behandlung des Zerfalls Jugoslawiens bei den Vereinten Nationen vergleichbar, wo alle Staaten, die aus der Dismembration Jugoslawiens hervorgegangen sind, eine neue UN-Mitgliedschaft beantragen mussten, während die Mitgliedschaft Jugoslawiens in den Vereinten Nationen erloschen ist.

Denkbar wäre es allerdings auch gewesen, dass die Mitgliedschaft beider Nachfolgestaaten in der EU fortbestanden hätte, woraufhin die notwendigen Anpassungen der Verträge bona fide innerhalb des Rechtsrahmens des Unionsrechts aus einer Mitgliedsposition heraus von den Vertragsparteien hätten verhandelt werden müssen. ${ }^{20}$ Hierfür könnte zunächst sprechen, dass diese Lösung dem Rechtsgedanken des Art. 34 der Wiener Konvention über das Recht der Staatennachfolge entspricht, die allerdings weder vom Vereinigten Königreich noch von einer Mehrheit der anderen EU-Mitgliedstaaten unterzeichnet wurde. Nach wohl richtiger Ansicht stellt Art. 34 der Konvention auch keine Kodifizierung eines

20 Vgl. Vaubel, The political economy of secession in the European Union v. 9.4.2013, www.vwl.uni-mannheim.de/vaubel/pdf-Dateien/ThePoliticalEconomy09.04.13.pdf (1.10.2014). 
allgemeinen völkerrechtlichen Grundsatzes dar, der auch ohne Ratifikation durch die betroffenen Staaten Gültigkeit beanspruchen könnte. ${ }^{21}$ Der Rechtsgedanke, dass notwendig werdende Vertragsanpassungen von den Vertragsparteien eines an sich bestehen bleibenden Vertrags bona fide zu führen sind, dürfte spezifisch für die EU-Verträge im Grundsatz der loyalen Zusammenarbeit zwischen der Union und ihren Mitgliedstaaten, Art. 4 Abs. 3 EUV, enthalten und damit anders als im allgemeinen Völkerrecht sogar ausdrücklich kodifiziert sein.

Für die Lösung fortbestehender Mitgliedschaft beider Nachfolgestaaten im Hinblick auf den schottischen Fall hätte weiter sprechen können, dass der EuGH, vergleichbar dem EGMR hinsichtlich der Geltung der Rechte aus der EMRK im Fall der Unabhängigkeit Montenegros, ${ }^{22}$ möglicherweise gewillt gewesen sein könnte, aus der Unionsbürgerschaft das Verbot herzuleiten, dass die unionsbürgerschaftlichen Rechte den Unionsbürgern einfach entzogen werden können. Zudem spricht die historische Erfahrung bei der Wiedervereinigung Deutschlands durch den Beitritt der fünf neuen Bundesländer zum Gebiet des EG-Mitgliedstaats Bundesrepublik Deutschland für die Möglichkeit der Anpassung der Mitgliedschaftsrechte innerhalb des Unionsrechtsrahmens.

Die fünf neuen Bundesländer sind zunächst dem Gebiet der Bundesrepublik Deutschland und damit der Europäischen Gemeinschaft beigetreten, ohne dass unmittelbar eine Anpassung des repräsentativen Gewichts Deutschlands erfolgt wäre, die erst im Rahmen einer größeren Vertragsreform nach bona fide geführten Verhandlungen innerhalb des mitgliedschaftlichen Rahmens nachgeholt wurden - auch wenn im deutschen Fall wegen der Vergrößerung des Bundesgebiets die zeitliche Verzögerung der Anpassung des repräsentativen Gewichts Deutschlands in den EG-Institutionen, insbesondere im Europäischen Parlament, sich lediglich für Deutschland selbst nachteilig ausgewirkt hat. In Analogie zum Vorgehen bei der deutschen Wiedervereinigung wäre es im hypothetischen britischen Fall allerdings denkbar gewesen, dass die beiden Nachfolgestaaten zunächst verpflichtet worden wären, die britischen Mitwirkungsrechte gemeinsam auszuüben, ${ }^{23}$ was allerdings eine erhebliche Disziplin der beiden Nachfolgestaaten erfordert hätte, die in einer emotionalen Situation, wie nach einem erfolgreichen Unabhängigkeitsreferendum, nicht unbedingt hätte vorausgesetzt werden können.

\section{Rechtslage bezüglich einzelner Mitgliedstaaten des Euroraums}

Unter den derzeit achtzehn Mitgliedstaaten des Euroraums, die gleichzeitig Mitgliedstaaten der Europäischen Union sind, bestehen Unabhängigkeitsbestrebungen vor allem in Spanien in der autonomen Region Katalonien, wo wie oben erwähnt am 9. November 2014 eine Volksabstimmung durchgeführt werden soll, deren Legalität noch fraglich ist. Unabhängigkeitsbestrebungen in Spanien existieren aber eventuell auch im Baskenland, in Galizien und einigen anderen Gebieten. Andere Mitgliedstaaten sind weniger mit dem Problem einer möglichen Unabhängigkeit als vielmehr mit einer drohenden Dismembration konfrontiert,

21 Zimmermann, (Fn. 15), S. 827.

22 EGMR, Nr. 11890/05, Bijelić/Montenegro und Serbien, insbesondere Rdnr. 69.

23 So insbesondere Vaubel, (Fn. 20). 
so vor allem Belgien. Starke regionale Identitäten zeigen jedoch, dass eine vorsorgliche Befassung mit dem Rechtsrahmen einer eventuellen Staatennachfolge auch für den Euroraum Sinn macht. Allerdings ist bei keinem der innerhalb des Euroraums möglicherweise in Zukunft relevant werdenden Fälle eine der - nach einer Interpretation - schottischen Besonderheit vergleichbare Situation gegeben, in der ein Mitgliedstaat als Union aus früher selbständigen Staaten, die ihre rechtliche Identität bewahrt haben, der Europäischen Union beigetreten ist.

\section{Katalonien als heutige spanische autonome Region}

Insbesondere Katalonien ist als heutige spanische autonome Region weder hinsichtlich der Institutionen noch territorial identisch mit dem Königreich Aragon, das durch die Hochzeit der katholischen Könige Ferdinand von Aragon und Isabella von Kastilien im Jahr 1469 in der Personalunion der spanischen Monarchie aufgegangen ist, auch wenn die katalanische Kultur das Königreich Aragon wesentlich geprägt haben mag. Insoweit besteht mit Blick auf Katalonien ein wesentlicher Unterschied zu Schottland, dessen territoriale und zumindest anfänglich auch institutionelle Integrität seit den Acts of Union fortbestand.

\section{Belgien}

Auch das Königreich Belgien erlebt eine Föderalisierung erst seit dem Jahr 1945, während es vorher weder flämische noch wallonische klar abgegrenzte staatliche Einheiten gab, sondern ein moderner Staat lediglich insgesamt als Königreich Belgien entstanden ist. Der Konflikt zwischen den Sprachgemeinschaften des Landes entstand somit erst in der belgischen Monarchie und geht ihr nicht voraus. Die von der flämischen Partei N-VA in Anbetracht der Ablehnung einer Unabhängigkeit Flanderns in der flämischen Mehrheitsbevölkerung vorgetragenen Pläne zum Umbau des Königreichs zu einer „Konföderation Belgien“ ${ }^{24}$ würden demzufolge dazu führen, dass diese Konföderation als fortbestehender Nationalstaat weiter Mitgliedstaat der Europäischen Union bliebe und sich die Landesteile Flandern und Wallonien im Rahmen der von ihnen zu findenden innerstaatlichen Aufgabenverteilung hinsichtlich ihrer Vertretung in den Organen der Europäischen Union (Europäischer Rat, Ministerrat, EZB-Rat) auf ein geeignetes Modell einigen müssten. Vom europäischen Blickwinkel aus würde sich in diesem Fall an der EU-Mitgliedschaft Belgiens als Nationalstaat nichts ändern. Es läge insofern nur eine Verschärfung der seit geraumer Zeit zu beobachtenden Verselbständigung der verschiedenen Landesteile vor.

24 Hierzu Stabenow, Ein Torso namens Belgien, FAZ v. 4.11.2013. 


\section{Innerhalb des Euroraums derzeit keine Konstellation denkbar, in der zwei Nachfolgestaaten gleichzeitig eine fortbestehende EU-Mitgliedschaft evozieren könnten}

Alle denkbaren Fälle von neuen Staatenbildungen im Euroraum bilden somit Situationen $a b$, in denen sich entweder eine Region für unabhängig erklärt, während der verbleibende Rumpfstaat in verkleinerter Form fortbestehen würde, oder in denen ein Staatswesen sich vollständig auflöst und durch zwei oder mehrere neue Staaten ersetzt würde.

Auf diese Fälle trifft jeweils eindeutig die von EU-Kommissionspräsident Barroso in seinem Brief an Lord Tugendhat geäußerte Einschätzung zu, dass diese neuen staatlichen Entitäten zunächst nicht eigenständig Mitglieder der Europäischen Union wären und die EU-Verträge folglich auf ihrem Territorium keine Geltung beanspruchen können. Für Katalonien hat der Präsident des Europäischen Rates, Herman Van Rompuy, am 12. Dezember 2013 auf diese Rechtslage anlässlich einer Pressekonferenz mit dem spanischen Ministerpräsidenten Rajoy ausdrücklich hingewiesen. ${ }^{25}$ Wie schon bei der Betrachtung Schottlands angesprochen, ist zudem Art. 34 der Wiener Konvention über das Recht der Staatennachfolge, der eine uneingeschränkte Kontinuität vorsehen würde, mangels Ratifikation durch alle Mitgliedstaaten der Europäischen nicht anwendbar und drückt auch keinen als allgemeinen Grundsatz des Völkerrechts universell akzeptierten Rechtsgedanken aus.

\section{Währungsrechtliche Konsequenzen}

Unter den gegenwärtig 28 Mitgliedstaaten der Europäischen Union trifft die rechtliche Verpflichtung zur Einführung des Euro bei Vorliegen der Voraussetzungen lediglich das Vereinigte Königreich und Dänemark nicht, da diese Länder ein entsprechendes Opt-out von der primärrechtlichen Verpflichtung erfolgreich verhandeln konnten. Dies entspricht dem Umstand, dass die Zustimmung beider Mitgliedstaaten zu den Änderungen der Unionsverträge, mit denen unter anderem die Regelungen zum Euro eingeführt wurden, sonst wohl ausgeblieben wäre und aufgrund der von Art. 48 Abs. 4 EUV für Vertragsänderungen erforderlichen Ratifikation in allen Mitgliedstaaten sonst wohl die Vertragsänderung an sich gescheitert wäre. Eine solche Blockadeposition fehlt hingegen einem Beitrittskandidaten im Beitrittsverfahren.

Hinsichtlich der Situation der restlichen Mitgliedstaaten ist zu unterscheiden, ob diese den Euro bereits eingeführt haben. In diesem Fall liegt die ausschließliche Zuständigkeit für die Währungspolitik gemäß Art. 3 Abs. 1 lit. c) AEUV bei der Union. Für Mitgliedstaaten mit Ausnahmeregelung im Sinne von Art. 139 Abs. 1 AEUV, also solche Mitgliedstaaten, für die der Rat nicht beschlossen hat, dass sie die erforderlichen Voraussetzungen für die Einführung des Euro erfüllen, liegt die Zuständigkeit für die Währungspolitik hingegen mit gewissen Einschränkungen ${ }^{26}$ grundsätzlich weiter auf der mitgliedstaatlichen Ebene.

25 EUCO 267/13 v. 12.12.2013.

26 Insbesondere ist gemäß Art. 142 AEUV die Wechselkurspolitik als seine Angelegenheit von gemeinsamem Interesse zu betrachten. 


\section{Das Vereinigte Königreich als Mitgliedstaat der EU, dessen Währung nicht der Euro ist und der rechtlich nicht zur Einführung des Euro verpflichtet ist}

Die Konsequenzen für das nach dem schottischen Votum für einen Verblieb im Vereinigten Königreich hypothetische währungsrechtliche Regime in einem unabhängigen Schottland und in einem verkleinerten Vereinigten Königreich ergeben sich zumindest zum Teil aus der Beantwortung der Vorfrage über eine fortdauernde oder neu zu begründende Mitgliedschaft in der EU.

\section{Währungsrechtliche Konsequenzen bei Verlust der Mitgliedschaft in der EU}

Geht man davon aus, dass im Zuge der Unabhängigkeit Schottlands entweder Schottland allein oder beide Nachfolgestaaten des Vereinigten Königreichs die Mitgliedschaft in der EU verloren hätten, so verbleibt die währungspolitische Kompetenz bei den jeweiligen unabhängigen Staaten, ohne vom Unionsrecht beeinflusst zu werden. Ein verkleinertes Vereinigtes Königreich hätte also allein das Pfund Sterling beibehalten oder in eine „Sterling-Union" mit dem unabhängigen Schottland eintreten können. Ein unabhängiges Schottland als Drittstaat, der alleine außerhalb der EU gestanden wäre, hätte sich zudem entscheiden können, eine eigene Währung einzuführen oder - vergleichbar der Situation in Montenegro - für eine einseitige Euroisierung optieren können, allerdings ohne damit eine Vertretung im Eurosystem oder Einfluss auf die Geldpolitik des Euroraums zu erhalten.

\section{Währungsrechtliche Konsequenzen bei einem Wiedereintritt in die EU nach zwischenzeitlichem Ausscheiden}

Die schottische Regierung und der ihrer Stellungnahme zugrunde liegende Bericht zu einem makroökonomischen Rahmenwerk für Schottland stellten zudem die Frage der Einführung des Euro in Schottland als eine freiwillige Möglichkeit dar und stützten sich dabei darauf, dass die als Beitrittsbedingung zum Euro geforderte zweijährige Zugehörigkeit zum Wechselkursmechanismus II freiwillig sei, woraus sich auch die Freiwilligkeit der Einführung des Euro ergebe. ${ }^{27}$ Diese Rechtsansicht stellt sich jedenfalls für die bestehenden Mitgliedstaaten der Union, die kein Opt-out vereinbart haben, als nicht zutreffend heraus. Für sie besteht eine primärrechtliche Verpflichtung zur Einführung des Euro. Denn der Euro stellt sich als ein Bestandteil des unionsrechtlichen acquis dar, zu dessen Übernahme sich die Mitgliedstaaten im Rahmen ihres Beitrittsvertrages verpflichtet haben.

Die schottische Regierung und die sie beratende Expertenkommission scheinen zudem davon ausgegangen zu sein, dass die Regierung und die Zentralbank eines verkleinerten Vereinigten Königreichs gewillt gewesen wären, mit einem unabhängigen Schottland über die Bildung einer „Sterling-Union“ zu verhandeln und in eine solche einzutreten, ohne die Frage zu problematisieren, ob die Bildung einer solchen „Sterling-Union“ überhaupt mit den Verpflichtungen eines EU-Mitgliedstaats - des verkleinerten Vereinigten Königreichs

27 Scottish Government, (Fn. 5), S. 26 und 223; Scottish Government, Fiscal Commission Working Group - First Report, Februar 2013, S. 127, Pkt. 7.37. 
oder des neuen schottischen Staats nach erfolgter Aufnahme in die EU - vereinbar gewesen wäre oder nur von Staaten außerhalb der EU hätte gebildet werden können.

Mit einer wegfallenden EU-Mitgliedschaft wäre allerdings auch das vom Vereinigten Königreich vereinbarte vertragliche Opt-out bezüglich der Verpflichtung zur Einführung des Euro entfallen. ${ }^{28}$ Im Zuge eines eventuellen neuen Beitrittsverfahrens nach Art. 49 EUV hätte sich daher weder ein unabhängiges Schottland noch ein verkleinertes Vereinigtes Königreich, das nicht die bisherige Unionsmitgliedschaft des aktuellen Vereinigten Königreichs fortgesetzt hätte, auf das bisherige Opt-out berufen können. ${ }^{29}$

Fraglich wäre auch gewesen, ob es im Rahmen solcher Beitrittsverhandlungen gelungen wäre, ein erneutes Opt-out zu verhandeln. Hierauf hat sich die EU bislang bei keinen Beitrittsverhandlungen - weder bei der Norderweiterung um Schweden, Finnland und Österreich im Jahr 1995 noch im Rahmen der Osterweiterung 2004 oder beim kroatischen Beitritt 2013 - eingelassen. Nach den Zielen der Union, die nach Art. 3 Abs. 4 EUV die Errichtung einer Wirtschafts- und Währungsunion vorsehen, deren Währung der Euro ist, dürften Verhandlungen über ein entsprechendes $O p t$-out, wie es dem Vereinigten Königreich und Dänemark gewährt wurde, mittlerweile nicht nur auf faktische, sondern gleichfalls auf erhebliche rechtliche Hürden stoßen. Denn die gemeinsam festgelegten Ziele der Union stellen einen fundamentalen Teil des acquis communautaire dar. Gelingt die Vereinbarung eines Opt-outs nicht, so sind daher generell nach Abschluss der Beitrittsverhandlungen die neuen Mitgliedstaaten zur vollständigen Übernahme des acquis verpflichtet. Dieser beinhaltet jedenfalls die rechtliche Verpflichtung zur Einführung des Euro bei Vorliegen der Konvergenzkriterien. Diese rechtliche Verpflichtung besteht auch dann fort, wenn die EUKommission kein erkennbares Interesse zeigt, bei einem Verstoß ein Vertragsverletzungsverfahren einzuleiten.

\section{Währungsrechtliche Konsequenzen einer fortbestehenden Mitgliedschaft eines verkleinerten Vereinigten Königreichs in der EU}

Geht man davon aus, dass ein verkleinertes Vereinigtes Königreich die Unionsmitgliedschaft des bisherigen Vereinigten Königreichs kontinuierlich fortgesetzt hätte, so gälte der oben unter 1. und 2. dargestellte Befund nur für den hypothetischen neuen unabhängigen schottischen Staat. Das verkleinerte Vereinigte Königreich hätte sich allerdings im Rahmen der Fortsetzung seiner Mitgliedschaft der - sich letztlich aus seinem Beitrittsvertrag in Verbindung mit dem Grundsatz von Treu und Glauben ergebenden - Verpflichtung ausgesetzt gesehen, bona fide aus seiner Mitgliedsposition heraus mit den anderen vertragsschließenden Parteien der Unionsverträge, also mit den anderen Mitgliedstaaten, über eine Anpassung der Modalitäten der Mitgliedschaft zu verhandeln. ${ }^{30}$ Dies belegen historisch beispielsweise die Verhandlungen, die nach der deutschen Wiedervereinigung zwischen den vertragschließenden Parteien bona fide über die Anpassung der Modalitäten der da-

28 Ohne Bezugnahme auf das Vereinigte Königreich Athanassiou/Shaelou, EU Accession from Within?, Yearbook of European Law 2014, S. 10.

29 Für Schottland ausdrücklich Harvey, (Fn. 6), S. 429.

30 So ohne den Hinweis auf den Grundsatz von Treu und Glauben, hingegen sogar von dem möglichen Erfordernis eines „,complete redrafting“ ausgehend ibid., S. 414. 
maligen deutschen EG-Mitgliedschaft, insbesondere hinsichtlich der verhältnismäßigen Repräsentanz deutscher Mitglieder im Europäischen Parlament, geführt wurden.

Die zu verhandelnden Positionen hätten sich zunächst recht offensichtlich - wie im Fall der deutschen Wiedervereinigung erfolgt - auf eine Korrektur der Stimmengewichtung im Rat, die der Veränderung des Bevölkerungsgewichts und der Mehrheitsverhältnisse entspricht, vor allem mit Blick auf die für die Erlangung einer qualifizierten Mehrheit im Rat erforderlichen Stimmengewichte, sowie auf die Anpassung der Größe der Gruppe der vom jeweiligen Mitgliedstaat zu wählenden Parlamentsabgeordneten gerichtet. Allerdings hätte sich vor dem Hintergrund eines möglichen untechnischen „Wegfalls der Geschäftsgrundlage“ die eventuell gleichfalls im Verhandlungsweg zu lösende Frage gestellt, ob das vom Vereinigten Königreich verhandelte währungspolitische Opt-out hinsichtlich der Verpflichtung zur Einführung des Euro weiter Bestand hätte haben können. Dies wäre etwa dann möglicherweise nicht der Fall gewesen, wenn sich durch die Unabhängigkeit Schottlands das wirtschaftliche Gewicht des Vereinigten Königreichs dergestalt verringert hätte, dass nicht mehr davon hätte ausgegangen werden können, dass das verkleinerte Vereinigte Königreich ein derartiges Opt-out erfolgreich hätte verhandeln können. Die faktischen Chancen zur Beibehaltung des Opt-outs für ein verkleinertes Vereinigtes Königreich hätten meines Erachtens allerdings recht gut gestanden, da auch das deutlich kleinere Dänemark ein vergleichbares $O p t$-out verhandelt hat und zudem die britische Finanzindustrie, die einen nicht unwesentlichen Einfluss auf die britische Verweigerung der Übertragung der währungspolitischen Kompetenz auf die Unionsebene gehabt haben dürfte, im Süden Englands konzentriert ist, der im hypothetischen verkleinerten Vereinigten Königreich verblieben wäre.

Streitigkeiten zwischen dem hypothetisch verkleinerten Vereinigten Königreich und den übrigen Mitgliedstaaten im Zusammenhang mit den erforderlichen Anpassungen der Modalitäten der Mitgliedschaft hätten als Streitigkeiten zwischen Mitgliedstaaten im Zusammenhang mit dem Gegenstand der Verträge aufgrund eines Schiedsvertrags gemäß Art. 273 AEUV beim EuGH anhängig gemacht und von diesem verbindlich entschieden werden können. ${ }^{31}$

\section{Währungsrechtliche Konsequenzen einer fortbestehenden Mitgliedschaft beider Nachfolgestaaten des bisherigen Vereinigten Königreichs in der EU}

\section{a) Anpassung der Modalitäten der Mitgliedschaft}

Geht man schließlich davon aus, dass sowohl ein unabhängiges Schottland als auch ein verkleinertes Vereinigtes Königreich als Nachfolgestaaten des bisherigen Vereinigten Königreichs weiter EU-Mitgliedstaaten geblieben wären, so hätte sich die Frage der Anpassung der Modalitäten der Mitgliedschaft auch für das unabhängige Schottland gestellt. Hinzu wäre die Problematik getreten, dass dem unabhängigen Schottland und dem verkleinerten Vereinigten Königreich die Mitgliedsrechte des bisherigen Vereinigten König-

31 Zu den Voraussetzungen und zum Umfang der Zuständigkeit des EuGH gemäß Art. 273 AEUV vgl. Cremer, (Fn. 1), Art. 273 AEUV, Rdnr. 2. 
reichs zunächst - bis zum erfolgreichen Abschluss der Verhandlungen - gemeinsam zugestanden hätten, was zu erheblichen Abstimmungsschwierigkeiten zwischen den beiden Staaten hätte führen können. Hinsichtlich des währungspolitischen Opt-outs des Vereinigten Königreichs hätte sich allerdings insbesondere für ein unabhängiges Schottland die Frage eines diesbezüglichen „Wegfalls der Geschäftsgrundlage“ mit besonderer Schärfe gestellt. Auch sich hieraus ergebende Streitigkeiten hätten als Streitigkeiten zwischen Mitgliedstaaten im Zusammenhang mit dem Gegenstand der Verträge aufgrund eines Schiedsvertrags gemäß Art. 273 AEUV beim EuGH anhängig gemacht und von diesem verbindlich entschieden werden können.

\section{b) Eintritt in eine ,,Sterling-Union“}

Nur in dieser Konstellation einer kontinuierlichen EU-Mitgliedschaft beider Nachfolgestaaten des bisherigen Vereinigten Königreichs hätten sich unionsrechtliche Bindungen für beide Nachfolgestaaten ergeben und es wäre daher die Frage aufgetreten, ob der Eintritt eines unabhängigen Schottlands in eine „Sterling-Union“ mit einem verkleinerten Vereinigten Königreich mit dem Unionsrecht vereinbar gewesen wäre. Insbesondere für ein verkleinertes Vereinigtes Königreich hätte sich aufgrund der Bindung an den Grundsatz des unionsfreundlichen Handelns (Art. 4 Abs. 3 EUV) selbst bei Fortgeltung des eigenen währungspolitischen Opt-outs die Frage gestellt, ob es durch die Vereinbarung einer Währungsunion mit einem Mitgliedstaat, der an sich zur Einführung des Euro rechtlich verpflichtet wäre, nicht seine unionsvertraglichen Pflichten verletzt. Dies wäre nur dann nicht der Fall gewesen, wenn der währungspolitische Opt-out eindeutig auch für ein unabhängiges Schottland fortgegolten hätte, und dieser Fall hätte (anders als für das verkleinerte Vereinigte Königreich) aufgrund der geringeren wirtschaftlichen Bedeutung eines unabhängigen Schottlands möglicherweise nicht vorgelegen. Er hätte eindeutig dann nicht vorgelegen, wenn das unabhängige Schottland nach einer Sezession erst im Rahmen eines eigenständigen Beitrittsverfahrens nach Art. 49 EUV Unionsmitglied geworden wäre.

Die von der schottischen Regierung im Fall eines erfolgreichen Unabhängigkeitsreferendums politisch gewünschte „Sterling-Union“ mit dem verkleinerten Vereinigten Königreich hätte sich daher in Vereinbarkeit mit dem Unionsrecht nur dann realisieren lassen, wenn es dem unabhängigen Schottland gelungen wäre, ein dem Vereinigten Königreich vergleichbares währungspolitisches Opt-out zu vereinbaren. Wie bei der Analyse einer neu zu begründenden Mitgliedschaft in der EU gezeigt, dürften jedoch Verhandlungen über ein entsprechendes Opt-out nach den Zielen der Union, die gemäß Art. 3 Abs. 4 EUV die Errichtung einer Wirtschafts- und Währungsunion vorsehen, deren Währung der Euro ist, auf erhebliche rechtliche Hürden stoßen und faktisch daher fast ausgeschlossen sein.

Demzufolge lässt sich feststellen, dass eine „Sterling-Union“ zwischen den beiden hypothetischen Nachfolgestaaten des Vereinigten Königreichs bei für beide Nachfolgestaaten fortbestehender EU-Mitgliedschaft mit sehr hoher Wahrscheinlichkeit das von den EUVerträgen vorgesehene währungsrechtliche Regime verletzt hätte. Die von der schottischen Regierung gewünschte Vereinbarung einer „Sterling-Union“ bei gleichzeitiger eigenständiger EU-Mitgliedschaft Schottlands dürfte daher ohne eine vorherige Änderung des exis- 
tierenden unionsrechtlichen Rahmens bezüglich des währungsrechtlichen Regimes, die ein Einvernehmen aller Mitgliedstaaten erfordert hätte, rechtlich nicht umsetzbar gewesen sein.

Verstöße gegen den acquis durch die Aufnahme von Verhandlungen zu einer „SterlingUnion“" hätten von der EU-Kommission gegenüber einem Mitgliedstaat im Wege des Vertragsverletzungsverfahrens nach Art. 258 AEUV oder gegenüber einem Beitrittskandidaten durch das Einfrieren etwaiger Beitrittsverhandlungen geahndet werden können.

\section{Zerfallende Mitgliedstaaten des Euroraums oder sich unabhängig erklärende Teilregionen von Mitgliedstaaten des Euroraums}

Die konsequente Anwendung des gefundenen Ergebnisses würde dazu führen, dass sich unabhängig erklärende Teilregionen von Mitgliedstaaten oder zerfallende Mitgliedstaaten mit der Erlangung der staatlichen Unabhängigkeit der Teilstaaten auch dann aus der Europäischen Union ausscheiden müssten, wenn sie dem Euroraum angehören. ${ }^{32}$ Sie müssten sodann ein Beitrittsverfahren gemäß Art. 49 EUV beantragen und in diesem Beitrittsverfahren die Erfüllung der Kriterien zur Einführung des Euro nachweisen. Diese Kriterien wurden hingegen für einen anderen Fall normiert, nämlich für den Fall, dass ein Mitgliedstaat eine andere Währung als den Euro verwendet und dem Euroraum beitritt.

Folgeprobleme ergeben sich daher während des Zeitraums bis zum erfolgreichen Beitritt zur Union als eigenständiger Mitgliedstaat nicht nur hinsichtlich der Einräumung der Unionsbürgerrechte und Grundfreiheiten an die Bürger dieser neuen Staaten, insbesondere in Fällen, in denen die bisherigen Unionsbürger schon von ihrem Freizügigkeitsrecht Gebrauch gemacht haben, sondern gerade auch innerhalb des Euroraums mit Blick auf die währungsrechtlichen Konsequenzen.

So stellt sich unmittelbar nach der Erlangung der staatlichen Unabhängigkeit die Frage einer rechtlich - indem die hierfür zuständigen Organe des neuen unabhängigen Staats sie festgelegen - oder zumindest faktisch erfolgenden Euroisierung; in der Folge ergeben sich daraus dann die angerissenen Anschlussprobleme hinsichtlich einer Nutzung des Euro als Währung im Rahmen einer neu zu begründenden Mitgliedschaft in der Europäischen Union.

Gerade aufgrund der tiefen rechtlichen, wirtschaftlichen und politischen Integration der im Euroraum miteinander verbundenen Mitgliedstaaten der Europäischen Union erscheint ein solches Ergebnis eines automatischen Ausscheidens aus den Strukturen der Europäischen Union unbefriedigend. Kernregionen der europäischen Wirtschaft, wie sie die Cluster um Barcelona, Brüssel oder Mailand darstellen, dürften schon aufgrund ihrer engen Vernetzung mit anderen Wirtschaftsregionen ein Interesse an einem stabilen währungspolitischen Rahmen, wenn nicht sogar ein ausgesprochenes Interesse an einem Verbleib im Euroraum haben. Es kann daher begründet gemutmaßt werden, dass Unsicherheiten über den währungspolitischen Rahmen und die anwendbare Währung in dem neuen Staat bzw. deren Wechselkurs zu den wichtigen Leitwährungen, insbesondere zum Euro, die Wirtschaft in den betroffenen Regionen erheblich belasten würden.

32 So auch Athanassiou/Shaelou, (Fn. 28), S. 10. 


\section{Verhandlungsbasierte Lösung für neue Entität wahrscheinlich}

a) Mitgliedschaftsrechte in der EU

Dementsprechend dürften schon faktische Erwägungen dazu führen, dass jedenfalls in Friedenszeiten innerhalb des Euroraums selbst bei einem politischen Erfolg einer Unabhängigkeitsbewegung das Wirksamwerden der staatlichen Unabhängigkeit so lange hinausgeschoben würde, bis auf dem Verhandlungsweg ${ }^{33}$ eine befriedigende Lösung aller offenstehenden Fragen, insbesondere der Währungsfrage, gefunden werden kann. Der Rechtsgedanke, austrittsbezogene Fragen im Wege der Verhandlung zu lösen, ist für einen austrittswilligen Mitgliedstaat in Art. 50 Abs. 2 Satz 2 EUV normiert. Auch wenn diese Regelung auf sich unabhängig erklärende Teilregionen von Mitgliedstaaten oder zerfallende Mitgliedstaaten keine direkte Anwendung findet, kann ihr doch das Verhandlungsprinzip als ein Leitprinzip entnommen werden, das auch für die hier angesprochenen Fälle interessenadäquat ist und das für den Fall, dass die sich unabhängig erklärenden Teilregionen von Mitgliedstaaten oder aus zerfallenden Mitgliedstaaten hervorgehenden neuen Staaten in der Union zu verbleiben wünschen, mit der Aufnahme von Beitrittsverhandlungen verknüpft werden könnte.

Diese Verhandlungen könnten, um ein Ausscheiden aus der Union zu vermeiden, zu einem Zeitpunkt geführt werden, in dem die Wahrnehmung der Mitgliedschaftsrechte in der Union noch über den bisherigen Mitgliedstaat erfolgt, in dem also eine Unabhängigkeit beispielsweise zwar in einem Referendum beschlossen, aber noch nicht umgesetzt wurde. Dies würde es allerdings erforderlich machen, dass die Europäische Union das von Art. 49 EUV definierte Staatlichkeitskriterium als Voraussetzung für die Aufnahme von Beitrittsverhandlungen im Wege einer interessengeleiteten Interpretation auf Gebilde ausdehnt, die die staatliche Unabhängigkeit noch nicht erreicht haben und aufgrund dieser Tatsache aktuell noch Bestandteil eines Mitgliedstaats der Europäischen Union sind. ${ }^{34}$ Dies dürfte allerdings zu erheblichen Spannungen in den Fällen führen, in denen ein als EU-Mitgliedstaat verbleibender Rumpfstaat die Segregationsbewegungen kategorisch bekämpft hat.

Obwohl der Wortlaut von Art. 49 EUV die Eigenstaatlichkeit bereits zur Stellung eines Beitrittsantrags voraussetzt, lässt sich insofern vertreten, dass eine teleologische Reduktion des Staatlichkeitskriteriums dahingehend möglich sein müsste, dass der Zeitpunkt der Erlangung einer eigenen Staatlichkeit nach einem Referendum im Rahmen eines Unabhängigkeitsprozesses mit dem Zeitpunkt des Beitritts zur Europäischen Union als eigenständiger Mitgliedstaat zusammenfallen kann. Eine entsprechende Zustimmung eines als

33 Hofmeister, Was bedeutet Schottlands Unabhängigkeit für die Mitgliedschaft in der EU?, EuR 2013, S. 711 übersieht mit seiner Forderung, der europäische Gesetzgeber möge sich de lege ferenda der Thematik annehmen und ein beschleunigtes Annahmeverfahren kodifizieren (S. 720), dass die Kodifikation eines solchen Verfahrens nur im Wege einer ordentlichen Vertragsänderung gemäß Art. 48 EUV und damit in einem zeitlich aufwändigen Verfahren erfolgen könnte. Die Verhandlungen zu dieser hypothetischen Vertragsänderung dürften eher komplexer und damit deutlich langwieriger werden als konkrete Verhandlungen zu sich in einem einzelnen Fall stellenden Problemkomplexen.

34 Zum Staatlichkeitskriterium als Beitrittsvoraussetzung zur Europäischen Union Rötting, (Fn. 7), S. 93. 
Mitgliedstaat in der EU verbleibenden Rumpfstaats bleibt gemäß Art. 49 UAbs. 1 Satz 2 EUV aber Voraussetzung für die Einleitung des Beitrittsverfahrens, sodass gegen dessen Widerstand kein EU-Beitritt möglich erscheint.

\section{b) Währungsrechtliche Fragen}

Mit Bezug auf neue unabhängige Staaten, die bislang Mitgliedstaaten des Euroraums angehörten, stellen sich zudem währungsrechtliche Fragen. So bleibt offen, ob ein Territorium, das bislang aufgrund einer Mitgliedschaft in der Wirtschafts- und Währungsunion den Euro als Zahlungsmittel verwendete und das sich im Zuge der Verhandlungen über eine eigenständige Mitgliedschaft in der Europäischen Union wieder rechtlich zur Einführung des Euro verpflichten will, für eine Übergangszeit zur Einführung einer anderen Währung rechtlich gezwungen werden kann und faktisch gezwungen werden sollte. Diese Überlegung erschiene jedenfalls dann widersinnig, wenn man von einer durchgängigen Erfüllung der Konvergenzkriterien zur Einführung des Euro ausginge und eine andere Währung nur eingeführt werden müsste, um die Erfüllung des von Art. 140 Abs. 1 Satz 3 Spiegelstrich 3 AEUV geregelten Konvergenzkriteriums der Wechselkursstabilität zum Euro unter Beweis zu stellen. Neben einer einseitigen Euroisierung, die jedenfalls faktisch nicht zu verhindern sein dürfte, untersuchen Athanassiou und Shaelou hierzu die Option einer Euroisierung durch einen währungsrechtlichen Vertrag auf der Grundlage von Art. 219 Abs. 3 AEUV. ${ }^{35}$ Nach dem klaren Wortlaut dieser Norm steht die Option zum Abschluss währungsrechtlicher Verträge der Union aber nur gegenüber Drittstaaten und internationalen Organisationen zu; ohne Anpassung der EU-Verträge dürfte hierin somit keine Rechtsgrundlage für eine im Konsens mit der EU vereinbarte Verwendung des Euro in Mitgliedstaaten der Union mit Ausnahmeregelung im Sinne des Art. 139 Abs. 1 AEUV liegen.

Eine vergleichbare Problematik dürfte sich in der Zukunft parallel im EU-Beitrittsverfahren von Montenegro stellen, das den Euro einseitig als gesetzliches Zahlungsmittel festgelegt hat und so das Konvergenzkriterium der Wechselkursstabilität derzeit gegenstandslos macht. Die Unionsorgane haben ihre negative Sichtweise einer einseitigen Euroisierung von Beitrittskandidaten gegenüber Montenegro zwar deutlich gemacht, hieraus aber keine währungsrechtlichen Konsequenzen gezogen, sondern lediglich darauf hingewiesen, dass die einseitige Euroisierung Montenegros eine historische Folge der einseitigen Verwendung der D-Mark als gesetzliches Zahlungsmittel in Montenegro war und nicht mit Montenegros Beitrittsambitionen im Zusammenhang steht. ${ }^{36}$

35 Athanassiou/Shaelou, (Fn. 28), S. $34 \mathrm{f}$.

36 Commission Staff Working Document, Analytical Report accompanying the Communication from the Commission to the European Parliament and the Council: Commission Opinion on Montenegro's application for membership of the European Union, SEC(2010) 1334 final v. 9.11.2010, Kap. 3.17, S. 84. 


\section{c) Rechtfertigung einer Andersbehandlung von Mitgliedstaaten des Euroraums und von neuen unabhängigen Staaten, die den Euro bislang verwendet haben?}

Angesichts der erheblichen Defizite hinsichtlich der Einhaltung der Stabilitätskriterien der Mitgliedschaft in der Wirtschafts- und Währungsunion in den meisten Mitgliedstaaten stellt sich zudem die Frage, ob man Gebiete, die als Teil eines Mitgliedstaats schon Teil des gemeinsamen Währungsraumes waren, hinsichtlich der Erfüllung der Konvergenzkriterien fundamental anders behandeln könnte als wenn sie Teil des jeweiligen Mitgliedstaats geblieben wären. Hier wird gerade ein gesteigertes Interesse bestehen, den Euro als Währung zu behalten.

Diese unionsverfassungsrechtliche, aber auch politische Frage ist der für das Beitrittsverfahren zur Europäischen Union zu beantwortenden Frage vergleichbar, ob die Beitrittskriterien zur Europäischen Union dem Niveau entsprechen müssen, das gegenüber bereits existierenden Mitgliedstaaten angewendet wird, oder über diese Standards hinausgehen dürfen. ${ }^{37}$ Nach Ansicht des Verfassers sprechen die besseren juristischen Gründe für eine konsistente Anwendung eines identischen Schutzniveaus. ${ }^{38}$ Das setzt allerdings voraus, dass die EU-Kommission ihrer von Art. 17 Abs. 1 Satz 2 EUV zugewiesenen Rolle als Hüterin der Verträge gegenüber den EU-Mitgliedstaaten auch nachkommt.

Eine eventuell so wahrgenommene Nachsichtigkeit der EU-Kommission gegenüber als vertragsbrüchig empfundenen Mitgliedstaaten könnte daher dazu führen, dafür zu plädieren, jedenfalls im Beitrittsverfahren striktere Standards anzuwenden und ein höheres Schutzniveau zu fordern. Diese Frage dürfte im Ergebnis für das Beitrittsverfahren nicht justiziabel sein und muss dort daher anwendungsbezogen gelöst werden. Für die hier behandelte währungsrechtliche Problematik ergäbe sich, solange die Mitgliedschaft in der Union durch ein Wirksamwerden der Unabhängigkeit ohne eine Aufnahme als eigenständiger Mitgliedstaat nicht beendet ist, hingegen zumindest dem Grundsatz nach die Möglichkeit, die offenen Fragen als Streitigkeiten zwischen Mitgliedstaaten im Zusammenhang mit dem Gegenstand der Verträge aufgrund eines Schiedsvertrags gemäß Art. 273 AEUV dem EuGH zur Entscheidung vorzulegen.

Fraglich ist allerdings, ob eine der beteiligten Parteien eine solche Streitentscheidung durch den EuGH tatsächlich wünschen würde. Im Fall eines Erfolgs eines Unabhängigkeitsreferendums im Euroraum dürfte daher wohl eher ein deutlicher Schwerpunkt auf der Suche einer verhandlungsbasierten Lösung liegen, der alle beteiligten Parteien zustimmen können. Einer verhandlungsbasierten Lösung entspricht auch der Rechtsgedanke aus Art. 50 EUV, der selbst Mitgliedstaaten, die aus der Union austreten wollen, einem Verhandlungsprozess unterwirft, ${ }^{39}$ sodass auch im Falle des drohenden Ausscheidens eines Teilgebiets eines Mitgliedstaats jedenfalls Verhandlungen über die Modalitäten geboten

37 Hierzu Rötting, (Fn. 7), S. 156 ff. Beispielsweise im Minderheitenschutz wurde diese Frage im kroatischen Beitrittsverfahren relevant, weil die minderheitenschutzrechtlichen Standards, die von der Republik Kroatien im Zuge ihres Beitrittsverfahrens implementiert werden sollten, innerhalb der Europäischen Union - insbesondere in Frankreich - nicht eingehalten wurden.

38 Hierzu für das Beitrittsverfahren ausführlich ibid., S. 175 ff. und $163 \mathrm{ff}$.

39 Hofmeister, (Fn. 33), S. 719. 
erscheinen könnten. ${ }^{40}$ Diese Verhandlungen könnten sich sehr lange hinziehen, sodass zwischen einem erfolgreichen Unabhängigkeitsreferendum und dem tatsächlichen Wirksamwerden der staatlichen Unabhängigkeit eines Gebiets mehrere Jahre liegen könnten, in denen das Gebiet weiter über seinen bisherigen Mitgliedstaat in den Institutionen der EU und des Eurosystems repräsentiert werden müsste, was aber politisch geradezu ein Paradoxon darstellt.

\section{Auswirkungen auf verkleinerte Rumpfstaaten}

Sofern ein Rumpfstaat nach einem erfolgreichen Unabhängigkeitsreferendum fortbesteht, stellt sich zunächst für diesen Rumpfstaat die Frage, ob er eine kontinuierliche Fortsetzung seiner Mitgliedschaftsrechte in der Wirtschafts- und Währungsunion beansprucht und beanspruchen kann, oder ob er gleichfalls als neuer Staat angesehen werden müsste, der die Mitgliedschaft in der Union erneut beantragen müsste. Damit verknüpft ist nämlich die währungsrechtliche Problematik, ob der verbleibende Rumpfstaat seine Mitgliedschaftsrechte in der Wirtschafts- und Währungsunion fortsetzen kann oder ob diese Mitgliedschaftsrechte erst neu begründet werden müssten, was insbesondere im Hinblick auf die wirtschaftliche Konvergenz äußerst problematisch werden könnte.

Bejaht man die erste Frage und vertritt, dass ein verbleibender Rumpfstaat seine Mitgliedschaftsrechte in der Europäischen Union im Allgemeinen und in der Wirtschafts- und Währungsunion in Besonderen fortsetzen kann, so müsste dieser verbleibende Rumpfstaat lediglich bona fide mit den verbleibenden Vertragsparteien Verhandlungen über die primärrechtliche Anpassung des Rahmens seiner Mitgliedschaft durchführen, insbesondere also im allgemeinen Unionsrecht über eine Anpassung der Stimmgewichtungen und der Zahl der ins Europäische Parlament entsandten Abgeordneten. Hinsichtlich der währungsrechtlichen Problematik ergibt sich der Bedarf einer Anpassung des Kapitalanteils der den verbleibenden Rumpfstaat im Europäischen System der Zentralbanken (ESZB) repräsentierenden Zentralbank an der Europäischen Zentralbank (EZB). Fraglich wäre hierbei insbesondere, ob diese Anpassung im Rahmen der regelmäßigen Anpassungen der den nationalen Zentralbanken zugeteilten Gewichtsanteile nach Art. 29.3 der Satzung des ESZB und der EZB erfolgen müsste, oder ob eine entsprechende Anwendung des Art. 48.3 der Satzung, der Anpassungen des EZB-Kapitalschlüssels bei Beitritten zur EU vorsieht, vorzunehmen wäre. Der Schlüssel für die Zeichnung des Kapitals der EZB ergibt sich in beiden Fällen aus Art. 29.1 der Satzung und greift jeweils hälftig die Bevölkerungsgröße des Mitgliedstaats und seine am Bruttoinlandsprodukt gemessene wirtschaftliche Leistungsfähigkeit auf. Die zur Anwendung des Art. 29.1 der Satzung zu verwendenden statistischen Daten werden von der Kommission gemäß Art.29.2 der Satzung nach den Regeln bereitgestellt, die der Rat (Ministerrat) nach dem Verfahren des Art. 41 der Satzung festlegt, der seinerseits auf Art. 129 Abs. 6 AEUV verweist. Für eine entsprechende Anwendung des Art. 48.3 der Satzung spricht die Vergleichbarkeit zur Beitrittssituation, während für die regelmäßige, alle fünf Jahre erfolgende Anpassung sprechen könnte, dass in einer Übergangsphase zwischen einem Unabhängigkeitsreferendum und dem Wirksamwerden 
der Unabhängigkeit der verbliebene Rumpfstaat ohnehin weiter den sich im Unabhängigkeitsprozess befindlichen Gebietsteil gegenüber den Unionsorganen repräsentieren müsste.

Für den verbliebenen Rumpfstaat dürften sich damit vor allem technische Fragen stellen, etwa hinsichtlich der Erfassung der erforderlichen statistischen Daten, wenn man davon ausginge, dass die bisherige Mitgliedschaft vom verbleibenden Rumpfstaat mit den erforderlichen Anpassungen unverändert fortgesetzt wird.

Anders stellt sich die Bewertung dar, wenn man davon ausgeht, dass ein verbleibender Rumpfstaat seine Mitgliedschaftsrechte nicht fortsetzen kann. Dieser Fall wäre für den jeweiligen verbleibenden Rumpfstaat den Fällen einer vollständigen Dismembration eines Mitgliedstaats sowie der Unabhängigkeit eines neu entstandenen Staats vergleichbar.

Daher ist davon auszugehen, dass in allen hypothetischen Fällen einer Unabhängigkeit, so insbesondere in einem verkleinerten Spanien bei einer hypothetischen Unabhängigkeit Kataloniens, die Entscheidungsträger des verbliebenen staatlichen Gebildes sich auf den Standpunkt stellen werden, dass ihr Staat die Mitgliedschaftsrechte in der Europäischen Union unverändert wahrnimmt.

\section{Auswirkungen des Ausscheidens eines EZB-Ratsmitglieds oder dessen Unvermögens, an einer Abstimmung teilzunehmen, auf die Beschlussorgane der EZB}

Eine bedeutsame Frage ist weiter die Funktionsfähigkeit der Beschlussorgane der EZB, die gemäß Art. 8 des Protokolls über die Satzung des Europäischen Systems der Zentralbanken und der Europäischen Zentralbank das ESZB leiten. Zwar beschließt insbesondere der EZB-Rat im Regelfall nach Art 10.2 der Satzung mit einfacher Mehrheit. Die Präsidenten der nationalen Zentralbanken des Eurosystems gehören dem EZB-Rat zudem ex officio an und sind gerade keine Vertreter nationaler Interessen. ${ }^{41}$ Daher würde ein Ausscheiden eines Ratsmitglieds oder dessen Unvermögen, an einer Abstimmung teilzunehmen, in der Regel nicht die Beschlussfähigkeit des EZB-Rats unterbinden und bliebe daher ohne Konsequenzen.

Allerdings sieht Art. 10.3 der Satzung auch Fälle vor, in denen eine gewichtete Abstimmung erfolgt. Hierbei geht es um Fragen, die die finanzielle Struktur des ESZB betreffen und sich finanziell auf die nationalen Zentralbanken als Kapitaleigner der EZB auswirken können. ${ }^{42}$ Für wichtige Fälle ist insoweit sogar eine qualifizierte Mehrheit von zwei Dritteln der gewogenen Stimmen erforderlich. Daher kann der Frage, ob ein bisheriges Mitglied des EZB-Rats in Folge von territorialen und politischen Veränderungen auf dem Gebiet seines bisherigen Mitgliedstaats überhaupt weiter stimmberechtigt ist und, falls ja, mit welchem Gewicht seine Stimmabgabe zu wiegen ist, durchaus eine erhebliche Relevanz zuwachsen. Festzustellen bleibt in diesem Zusammenhang, dass diese Rechtsfrage sich nur auf die Behandlung verkleinerter Rumpfstaaten bezieht, während sich unabhängig erklärende Teilregionen von Mitgliedstaaten des Euroraums oder neue staatliche Entitäten, die

41 Steven, in: Siekmann (Hrsg.), Kommentar zur Europäischen Währungsunion, 1. Aufl. 2012, Art. 10 der Satzung des ESZB und der EZB, Rdnr. 7.

42 Ibid., Rdnr. 39. 
aus zerfallenden Mitgliedstaaten hervorgegangen sind, selbst dann keine Repräsentanz im EZB-Rat und damit keinen Einfluss auf die Geldpolitik der EZB erhalten, wenn sie sich für eine faktische Euroisierung entscheiden sollten. Denn hierfür ist neben einer bestehenden Mitgliedschaft in der Union Voraussetzung, dass die in Art. 140 Abs. 1 AEUV geregelten Voraussetzungen erfüllt sind und der Rat auf dieser Grundlage gemäß Art. 140 Abs. 2 AEUV die Ausnahmeregelung bezüglich des betreffenden Mitgliedstaats aufhebt. 